\title{
Die kulturelle Dynamik Brasiliens ${ }^{1}$
}

\author{
Horst Nitschack
}

Trotz seiner politischen und wirtschaftlichen Bedeutung hat Brasiliens Kultur auf der internationalen Bühne nicht die gleiche Verbreitung und dasselbe Ansehen wie die hispanoamerikanischen Kulturen erreicht. Ein wichtiger Grund hierfür ist sicher die Bedeutung der präkolumbianischen Kulturen - Tolteken und Azteken in Mexiko, Mayas in Zentralamerika und Inkas in den Anden -, für die es im Raum des heutigen Brasiliens keine Entsprechungen gab. Auch während der Kolonialzeit bis zu den Unabhängigkeitserklärungen der neuen amerikanischen Nationen war die kulturelle Ausstrahlung der hispanoamerikanischen Kolonien nach Europa von größerer Bedeutung als die des portugiesischen Kolonialreichs in Amerika. Dies lag nicht zuletzt an der portugiesischen Kolonialpolitik, die darauf abzielte, den riesigen Raum in direkter Abhängigkeit vom Mutterland zu halten. Die portugiesischen Kolonialherren gründeten keine Universitäten, die Eliten der Kolonie waren für ihre Ausbildung auf das Mutterland, vor allem auf die Universität in Coimbra, angewiesen. Die Einrichtung von Druckereien, die ein eigenständiges intellektuelles Leben ohne direkte Überwachung durch die Beamten der portugiesischen Krone gefördert hätten, war untersagt. Die Plantagenwirtschaft (zuerst Zucker, später Kakao und Kaffee) als der wichtigste wirtschaftliche Produktionszweig basierte vor allem auf der Ausbeutung aus Afrika importierter Sklaven und verlangte nur einen geringen Anteil an städtischen Eliten. Ganz anders in Hispanoamerika, wo die kolonialen Metropolen nicht nur Handelszentren, sondern auch Verwaltungszentren waren und gut ausgebildete Kolonialbeamte benötigten. Ein im Vergleich mit den spanischen Kolonien relativ geringes allgemeines Bildungsniveau im portugiesisch sprechenden Teil Amerikas war die Folge.

Die entscheidende Wende begann im Jahr 1808 mit dem Umzug des gesamten portugiesischen Königshauses und seines Hofstaates von Lissabon nach Rio de Janeiro. Im Gegensatz zum spanischen König Karl IV

1 Dieser Beitrag entstand im Zusammenhang des Forschungsprojektes FONDECYT Nr. 1120116. 
kapitulierte der portugiesische Prinzregent João VI nicht vor den napoleonischen Truppen, sondern entschloss sich, seinen Regierungssitz nach Rio de Janeiro zu verlegen und bestieg mit mehr als 10.000 Begleitern eine von den Engländern bereitgestellte Flotte. Dieser Zustrom an Politikern, Verwaltungsbeamten und der zugehörigen Infrastruktur bedeutete für die verschlafene Tropenstadt am Zuckerhut einen entscheidenden Wandel. Rio de Janeiro erhielt ein neues Theater und eine Bibliothek, in der die 70.000 Bände der Lissaboner Staatsbibliothek, die im königlichen Umzugsgepäck mitgeführt wurden, untergebracht werden konnten. Nach der endgültigen Niederlage Napoleons rief João VI 1816 die 'Französische Kunstmission' mit dem Auftrag ins Land, die künstlerische Ausbildung in Brasilien nach französischem Vorbild zu modernisieren. Ihr gehörten Namen an wie Jean-Baptiste Debret, Nicolas-Antoine und Auguste Marie Taunay, Vater von Félix Taunay. Wenn die Wirkung der Mission auch weit hinter den Erwartungen zurückblieb, die in sie gesetzt wurden, so kam mit ihr doch ein für die Herausbildung der brasilianischen Kultur entscheidender weiterer Faktor ins Spiel: die Rezeption und Aneignung der kulturellen Traditionen und künstlerischen Produktionen der anderen europäischen Länder, vor allem derjenigen Frankreichs. Auch die deutschsprachigen Länder spielten in den nächsten Jahrzehnten in der kulturellen und wissenschaftlichen Entwicklung des ab 1822 als Kaiserreich unabhängigen tropischen Staates eine nicht zu unterschätzende Rolle. 1817 traf in Begleitung von Maria Leopoldine von Österreich, der zukünftigen Gemahlin des Kaisers von Brasilien, eine Gruppe von österreichischen und deutschen Wissenschaftlern in Brasilien ein, unter anderem die Naturwissenschaftler Johann Baptist von Spix und Carl Friedrich Philipp von Martius. Sie leisteten zusammen mit einer Reihe weiterer deutscher Naturwissenschaftler und Anthropologen in der Folgezeit einen wichtigen Beitrag zur Erforschung der tropischen Natur Brasiliens. Zu ihnen gehörten Wilhelm Ludwig von Eschwege, Georg Heinrich Freiherr von Langsdorff, an dessen Expedition ins Innere Brasiliens (1824-28) auch zeitweise der Maler Johann Moritz Rugendas teilnahm und später dann Karl von der Steinen und Theodor Koch-Grünberg, die ihre bedeutenden Amazonas-Expeditionen in den 80er Jahren des 19. Jahrhunderts und zu Beginn des 20. Jahrhunderts durchführten. Wenn deren Forschungsreisen - vor allem die der beiden Letztgenannten - auch anthropologisch von großer Bedeutung waren, so wurde doch durch ihre Publikationen in Deutschland ein Brasilienbild verbreitet, das 
das Land vor allem mit den Kulturen der Indianerstämme der unzugänglichen Amazonasregionen identifizierte.

Ein solcher Exotismus war allerdings bereits von der brasilianischen Romantik selbst gefördert worden. Auf der Suche nach einer selbstständigen brasilianischen Kultur, die sich von der Kultur der ehemaligen Kolonialmacht absetzt, entdeckten Autoren wie Gonçalves de Magalhães, José de Alencar und Gonçalves Dias die indianischen Kulturen des 16. und 17. Jahrhunderts, deren Vertreter sie entweder nach dem Vorbild antiker Helden oder als Verkörperungen des Rousseau'schen 'edlen Wilden' in ihren Epen und Romanen auftreten ließen. Im einen Fall war der brasilianische Indianer ein tropischer Herkules oder eine tropische Artemis, im anderen Fall Brasilien der Ort, in dem der 'edle Wilde' tatsächlich existiert hatte. Im ersten Fall lag die Absicht zugrunde, die indianischen Stämme Brasiliens zu Vertretern von humanen Werten zu machen, die denen des antiken Griechenlands nicht nachstehen, im zweiten Fall wurde der Rousseau'sche 'edle Wilde' für Brasilien als Wirklichkeit postuliert. Beide Fälle können als Argumentationsstrategien der brasilianischen Eliten interpretiert werden, eine kulturelle Eigenständigkeit für Brasilien zu reklamieren, bei der das Land als unabhängige Nation den europäischen Kulturnationen auf Augenhöhe begegnete.

Die Künste, vor allem eine Nationalliteratur, waren für die neu entstehenden lateinamerikanischen Nationen von ähnlich großer Bedeutung wie einige Jahrzehnte zuvor für die europäischen Länder. Für die internationale Anerkennung als selbstständige Nation spielte die Existenz einer Nationalliteratur eine entscheidende Rolle, denn das Recht auf $\mathrm{Na}$ tion wurde mit dem Verweis auf eine bereits bestehende Nationalliteratur mit ihren Mythen, Sagen und Märchen legitimiert. Das galt auch im Falle Brasiliens. Der Literatur kam dabei nicht nur die Aufgabe der nationalen Integration zu, d. h. Landschaften, Schauplätze, Figuren, Konflikte und ganz allgemein Erzählungen zu erfinden, die Brasilien als Brasilien identifizierten und ein Bild von einem Land entstehen ließen, mit dem sich die Leser der schmalen Elite identifizieren konnten. Die Literatur war auch der privilegierte Ort und die Nationalliteratur die privilegierte Institution, in der eine eigene brasilianische Sprache gefordert und gefördert wurde. Sie setzte sich vom Portugiesisch der ehemaligen Kolonialmacht nicht nur in der Aussprache, sondern auch in Syntax und Lexik ab. Die Gründung der Brasilianischen Akademie der Literatur Ende des 19. Jahrhunderts (1896) war die logische Folge dieses Prozesses. Ihr ging die Veröffentli- 
chung der ersten brasilianischen Literaturgeschichte von Silvio Romero im Jahre 1888 voraus. Noch früher war bereits 1863 in Berlin von dem österreichischen Gelehrten Ferdinand Wolf die erste Geschichte brasilianischer Literatur auf Französisch veröffentlicht worden: Le Brésil littéraire. Histoire de la littérature brésilienne. Im Vorwort argumentierte Wolf, die brasilianische Literatur habe in den letzten 30 Jahren Fortschritte gemacht, die danach verlangten, ihr einen Platz unter den Nationalliteraturen einzuräumen, was implizit bedeutete, man müsse der jungen brasilianischen Nation auch ihren Platz unter den Nationen einräumen.

Wenn auch der Beitrag der ausländischen Wissenschaftler für die naturwissenschaftliche Erforschung Brasiliens entscheidend war, so entstand doch gleichzeitig eine eigene Wissenschaftstradition. 1838 wurde in Rio de Janeiro das Brasilianische Historische und Geographische Institut gegründet, das es sich zur Aufgabe machte, die historische, geographische und ethnische nationale Wirklichkeit Brasiliens zu erforschen und zu dokumentieren. In diesem Zusammenhang wurde dann im Laufe der zweiten Hälfte des 19. Jahrhunderts der Positivismus zur wichtigsten theoretischen Referenz für die brasilianischen Intellektuellen. Allerdings sah sich dessen Fortschrittsphilosophie mit einer philosophisch nicht zu rechtfertigenden nationalen Wirklichkeit konfrontiert: Jenes Brasilien, das sich mit seiner Unabhängigkeit im Jahre 1822 auf den Weg gemacht hatte, eine moderne Nation zu werden, war bis 1888 eine Gesellschaft, die von Sklavenarbeit lebte und die dem größten Teil der im Land lebenden Menschen keine Bürgerrechte zugestand. Bis 1865 befand sich Brasilien damit noch in 'guter Gesellschaft', denn bis zu diesem Zeitpunkt hatten auch die Vereinigten Staaten die Sklaverei noch nicht abgeschafft. Ab Mitte der 60er Jahre des 19. Jahrhunderts gerieten die brasilianischen Eliten jedoch unter einen doppelten Druck: Gleichzeitig mit der Sklavenbefreiung in den Vereinigten Staaten trat Brasilien zusammen mit Uruguay und Argentinien in einen Krieg gegen Paraguay, ein Krieg, der sich über fünf Jahre hinziehen sollte und zu dem Brasilien auch auf die Unterstützung durch seine schwarze Bevölkerung angewiesen war. Das Resultat war das Gesetz der 'freien Geburt' von 1871, auf Grund dessen alle Kinder von Sklavinnen von nun an als Freie geborenen wurden.

Sowohl durch den Positivismus, für den die drei Faktoren 'Ort', 'Moment' und 'Rasse' für eine wissenschaftliche Beschreibung von Kulturen und damit für ihre Einordnung auf der Skala des Fortschritts entscheidend waren, wie auch durch die Rezeption des europäischen Rassismus, 
vor allem seiner Vertreter Gobineau und Buckle - Gobineau diente als französischer Diplomat in Rio de Janeiro und hatte direkten Zugang zum Hof Pedros II und zum Kaiser selbst - war das Paradigma 'Rasse' zu einer zentralen Kategorie zur Beschreibung von Kultur - im konkreten Fall der brasilianischen Kultur - geworden. Mehr noch, die Schwierigkeiten einer Nation, sich in der Geschichte des Fortschritts einen führenden Platz zu sichern, wurden als Konsequenz ihres rassischen Erbes und ihrer Rassenkonfiguration interpretiert. Konsequenterweise beschrieb Silvio Romero in seiner Literaturgeschichte Brasilien als eine Nation von drei Rassen und hatte keinen Zweifel daran, damit die Besonderheit seiner Kultur wissenschaftlich bestimmt zu haben. Sie war für ihn eine Kultur mit vor allem portugiesischen, aber auch einem gewissen europäischen Anteil, dem 'weißen' Element, dazu kamen indianische Elemente und der afrikanische kulturelle Anteil. Wenn seiner Ansicht nach die indianischen und afrikanischen Elemente für das Verständnis brasilianischer Kultur auch keinesfalls unterschlagen werden durften, so war er doch gleichzeitig davon überzeugt, der Weg zu einem modernen, zivilisierten Brasilien sei nur über eine 'Aufweißung' (blanqueamento) zu erreichen. Einen entscheidenden Beitrag dazu sollte die europäische Einwanderung leisten, die seit der zweiten Hälfte des 19. Jahrhunderts immer wieder durch staatliche Programme gefördert wurde. Diese Idee der 'Aufweißung' hatte trotz all ihrer Fragwürdigkeit einen für den brasilianischen kulturellen Diskurs wichtigen Nebeneffekt, der eine tiefgreifende Neudefinition des Rassenparadigmas zur Folge hatte. Zwar hatte der klassische Rassismus eines Gobineau mit der weißen Rasse an der Spitze eine Hierarchie der Rassen festgelegt, das zentrale ethnische Problem aber war ihm die Vermischung der Rassen, die mestizagem. Nach Gobineau zerstört die Rassenmischung den Wert, der jede Rasse - auch die inferioren - auszeichnet. Vor allem diese Vermischung sei für die Unfähigkeit Brasiliens verantwortlich, auf den Zug des Fortschritts aufzuspringen. Mehr noch, sie würde - so Gobineaus Prognose - unweigerlich in weniger als 200 Jahren zum Untergang des brasilianischen Volkes führen. Bereits mit Euclides da Cunhas Krieg im Sertão (1902) setzte eine folgenreiche Uminterpretation ein. Laut da Cunha war aus der Vermischung, die im Inneren des Landes bereits seit mehreren hundert Jahren am Werk war, eine neue Rasse entstanden: der Mensch des Sertão, der sich vor allem durch seine Stärke und seine Widerstandskraft auszeichnete. Vermischung führe also nicht notwendigerweise zum Untergang, sondern unter bestimmten Voraussetzungen 
finde gewissermaßen eine 'natürliche', darwinistische Zuchtauswahl statt. Wenn auch sein impliziter Rassismus da Cunha noch zwang, eine neue Rasse zu erfinden, so fand mit ihm doch zu Beginn des 20. Jahrhunderts eine entscheidende Wende statt: die Vermischung der Rassen wurde als Chance und nicht als Verhängnis angesehen. Der mexikanische Intellektuelle José Vasconcelos feierte in seinem in den 1920er Jahren in ganz Lateinamerika gelesenen Erfolgsbuch Die kosmische Rasse (1925) gerade die Vermischung aller großen Rassen in Lateinamerika, besonders aber in Brasilien, als die Hoffnung nicht nur Lateinamerikas, sondern der gesamten Menschheit.

1889 wurde die Republik der Vereinigten Staaten von Brasilien ausgerufen, eine Konsequenz der historischen und politischen Transformationsprozesse, deren deutlichster Ausdruck die Abschaffung der Sklaverei im Jahre zuvor war. Nicht nur der Name verriet die Vorbildrolle, die den im Laufe des 19. Jahrhunderts zur führenden hemisphärischen Macht avancierten Vereinigten Staaten von Amerika dabei zukam. Auch die erste Fahne der Republik war unübersehbar eine Imitation der US-amerikanischen 'Stars and Stripes', wenn auch in den brasilianischen Farben grün und gelb. Diese Fahne wurde kurz darauf durch die heute noch offizielle Nationalfahne ersetzt, in deren Zentrum die positivistische Parole 'Ordnung und Fortschritt' steht. Dieser Wechsel stand für die beiden gegensätzlichen Haltungen der brasilianischen Eliten. Die einen bewunderten den mächtigen nördlichen Nachbarn als Vorbild, während er für die anderen eine Bedrohung der brasilidade, des echten Braslianertums darstellte. Der Blick auf den Nachbarn hoch im Norden, ob ablehnend oder bewundernd, führte immer direkt oder indirekt zu der Frage, warum es Brasilien im Laufe des 19. Jahrhunderts, dem Jahrhundert des allgemeinen Fortschritts, nicht so weit gebracht habe wie die Vereinigten Staaten. War es das tropische Klima? War es die Natur mit ihren undurchdringlichen Wäldern, die jeden Kilometer konstruierter Straße oder Eisenbahnlinie zu einem menschenvernichtenden Abenteuer werden ließ? War es die rassische Konstellation, der zu geringe Anteil an weißer Bevölkerung, die Mentalität der afrikanischen Bevölkerung oder der hohe Anteil an Mulatten (Silvio Romero)? War es das koloniale, vor allem das portugiesische Erbe, das diesen Kolonisatoren im Vergleich mit ihresgleichen aus dem europäischen Norden, den Siedlern und Einwanderern in die USA, weniger Tüchtigkeit und Effizienz mit auf den Weg gab (Manoel de Bomfim, Paulo Prado)? War es die Religion, die puritanische Arbeitsmoral im 
Norden und der meridiane Katholizismus in Brasilien (Sérgio Buarque de Holanda)? Waren es die ungleichen Besitzverhältnisse, der Großgrundbesitz und die Plantagenwirtschaft mit ihren Heerscharen an besitzlosen Landarbeiten (Caio Prado Júnior)?

Zwischen dem Ende des 19. Jahrhunderts und bis in die 40er Jahre des 20. Jahrhunderts stellten brasilianische Schriftsteller und Wissenschaftler diese Frage ganz unterschiedlich und gaben dementsprechend auch unterschiedliche Antworten darauf. Was von den einen als Problem beschrieben wurde, interpretierten andere als Möglichkeit, als ein Potential für künftige Entwicklungen. Zuweilen fanden sich beide Positionen beim gleichen Autor: Euclides da Cunha interpretierte im Krieg im Sertão (1902) die Rückständigkeit des Nordostens, die Bereitschaft zum religiösen Fanatismus und zur Gewalt seiner Bewohner gleichzeitig als das Potential einer zukünftigen Rasse; für Oswald de Andrade war das indianische Element, das nach ihm jedem Brasilianer tief eingeschrieben ist, gerade der Garant dafür, dass Brasilien einen unverdinglichten und menschenfreundlichen Umgang mit den modernen Techniken finden werde (Das Antropophage Manifest, 1928); für Gilberto Freyre in Herrenhaus und Sklavenbütte (1933) entstand aus dem Jahrhunderte langen Zusammenleben von Portugiesen und Afrikanern auf den Zuckermühlen nicht nur eine Vermischung ihrer Kulturen, sondern eine neue Kultur, die brasilianische. Das heißt, die Vertreter dieser zweiten Gruppe waren sich einig - wenn auch mit ganz unterschiedlichen Argumenten -, der nördliche kapitalistische Weg könne nicht der Weg des tropischen Brasiliens sein.

Bei aller Vielfältigkeit der Fragen und der Antworten war ihnen aber allen der Blick auf dieses Land gemeinsam: als Sorge, als Hoffnung, als überschwängliches Lob (ufanismo). Welche wissenschaftliche Haltung, welche ideologische Überzeugung, welche Weltanschauung dem Diskurs auch zugrunde liegen mochte, ob sie pessimistisch, kritisch-zweifelnd, optimistisch oder in jubilierender Selbstbejahung vorgetragen wurde, eine grundsätzliche Infragestellung des 'Projekts Brasilien', seiner Einheit und seiner kulturellen Werte, fand sich nirgends. Alle waren auf der Suche nach einer gesellschaftlichen Ordnung, die es allen möglich macht, in diesem Land zu leben, und alle teilten das Gefühl, es gäbe etwas, was alle Brasilianer verbinde und sie zu Brasilianern mache. Was das sei, darin gingen die Antworten ähnlich auseinander wie bei der Frage, wer oder was für die fehlende Entwicklung verantwortlich sei oder was das besondere Entwicklungspotential Brasiliens ausmache. 
Hundert Jahre nach der Unabhängigkeit fand 1922 im Rahmen der zahlreichen Gedenkfeiern auch die Kunstwoche von São Paulo statt, mit Namen wie Oswald und Mário de Andrade, Menotti del Picchia, Plinio Salgado, Anita Malfatti, Heitor Villo-Lobos und einer Reihe weiterer bedeutender Künstler. Es war die brasilianische Avantgarde-Bewegung, die sich selbstbewusst präsentierte: vor dem eigenen brasilianischen Publikum, wie auch mit einem selbstbewussten Blick auf die in der Vergangenheit als Vorbild bewunderten europäischen Kulturen und Wissenschaften. Im Manifest Brasilholz (Pau Brasil) wurde zwei Jahre später das Programm auf den Begriff gebracht: Brasilien solle ein Land des Poesie-Exports und damit des Kulturexports werden und nicht nur Rohstoffe exportieren und die Kultur der entwickelten Länder importieren.

Das gab einer Entwicklung Ausdruck, die Brasilien in den Jahren seit der Gründung der Republik vollzogen hatte: Rio de Janeiro war in den letzten Jahren des 19. Jahrhunderts grundlegend modernisiert worden zum Teil gegen heftigen Widerstand der Bevölkerung. Die Elektrifizierung wurde vorangetrieben und Prachtstraßen angelegt, Tunnel durch die Berge getrieben, die die verschiedenen Stadtteile voneinander trennten, und eine Seilbahn auf den Zuckerhut hinauf konstruiert. 1920 hatte Rio de Janeiro mehr als eine Million Einwohner. In den Romanen von Machado de Assis - für viele Kritiker bis heute der bedeutendste Autor der brasilianischen Literatur - war dieses Rio de Janeiro noch als recht idyllische tropische Hauptstadt erschienen, wenn auch als scheinheilige und letztlich verlogene Idylle. Capitu, die Protagonistin aus Dom Casmurro (1899), wurde lange Zeit als eine Madame Bovary der Tropen gelesen. Erst in den 1960er Jahren wies die Literaturkritik darauf hin, dass der Leser im Roman keinen anderen Beleg für Capitus Ehebruch hat, als die paranoiden Phantasien ihres ewig missgelaunten Ehemanns Casmurro. Dieses scheinbar idyllische Rio de Janeiro mit seinen Stränden und dem Zuckerhut erfuhr mit der Deklaration der Republik einen tiefgreifenden Umbruch. Vor allem an der Peripherie, wo vorher die Sommerhäuser der Oligarchie und des wohlhabenden Bürgertums standen, entstanden jetzt die ärmlichen Siedlungen einer sich neu herausbildenden Gesellschaftsschicht mit ganz neuen Subjekten, eine Mischung aus Gelegenheitsarbeitern, ersten Ansätzen eines Proletariats und Kleinhändlern. Siedlungen, in denen sowohl aus den Migranten aus dem Nordosten wie auch aus befreiten ehemaligen Sklavinnen und Sklaven neue soziale Subjekte entstanden, die dann im Laufe des 20. Jahrhunderts immer mehr zu Akteuren in der brasiliani- 
schen Geschichte wurden. Mit Aluísio Azevedos naturalistischem Roman Cortiço (1890) sowie mit dem Roman O Mulato (1881) trat diese neue gesellschaftliche Wirklichkeit erstmals in die brasilianische Literaturszene.

Es waren Jahrzehnte eines rasanten Umbruches, in denen die Widersprüche der Modernisierung krass zutage traten: Die Kaffeeplantagen, die Brasilien zum größten Kaffeeexporteur der Welt werden ließen, lagen zum größten Teil im Bundesstaat São Paulo. Der Reichtum aus diesem Handel verwandelte die Stadt São Paulo in diesen Jahrzehnten in die modernste Stadt des Landes, einer der Gründe, weswegen die Kunstwoche der brasilianischen Avantgarde 1922 dort und nicht in Rio de Janeiro stattfand. Hunderttausende von europäischen Einwanderern, vor allem aus Italien und Deutschland, strömten in diesen Jahrzehnten in den Südosten und Süden Brasiliens und beeinflussten die Kultur dieser Regionen nachhaltig. Es wiederholte sich im Süden, was bereits Jahrzehnte zuvor im Norden aufgrund des Kautschuk-Booms passiert war, durch den noch nie gesehener Reichtum in das Amazonasgebiet gespült worden war. Für kurze Zeit hatte er Manaus zur modernsten Stadt Brasiliens mit elektrischer Beleuchtung und elektrischer Straßenbahn gemacht, in deren neu errichtetem Opernhaus (1896) Künstler aus ganz Brasilien und aus Europa auftraten. Im Gegensatz zum Kautschuk-Boom im Norden, der ab 1910 ein abruptes Ende fand, gelang es in São Paulo den durch den Kaffeeexport gewonnenen Reichtum in industrielles Kapital zu transformieren, die Grundlage dafür, dass diese Stadt im Laufe des 20. Jahrhunderts zu einer Megalopolis und zur größten Industriestadt Südamerikas wurde. Am Ende des Jahrhunderts verwandelte sich Brasilien nicht nur wirtschaftlich in einen global player, sondern es wurde auch als Kulturnation zu einem Exporteur, so wie es Oswald de Andrade in seinem Manifest Brasilholz gefordert hatte.

Die tiefgreifenden Veränderungen der brasilianischen Gesellschaft während des gesamten 20. Jahrhunderts fanden nicht nur in der Kultur ihren Ausdruck, gleichzeitig war es auch die Kultur, die diesen Wandel ermöglichte. Die Konsequenzen der Modernisierung waren Herausforderungen, mit denen Brasilien und seine Kultur im Laufe des 20. Jahrhunderts konfrontiert war. Zu ihnen gehörten die Industrialisierung und eine fortschreitende soziale Ausdifferenzierung, die Binnenmigration aus dem Nordosten in den Südosten und Süden des Landes (vor allem nach Rio de Janeiro und São Paulo), die Urbanisierung (heute leben ca. 80 \% der brasilianischen Bevölkerung in urbanen Agglomerationen), der Bedeutungsverlust persönlicher gesellschaftlicher Bindungen (Familien, Nach- 
barschaftsstrukturen, hierarchische Ordnungen) und der traditionellen Wertsysteme sowie die Entstehung einer den gesamten nationalen Raum organisierenden Marktgesellschaft und des damit verbundenen Individualisierungsprozesses.

Der Aufbau eines alle Bundesstaaten einbeziehenden Schulsystems ermöglichte trotz aller Defizite dem größten Teil der Bevölkerung eine Grundbildung, zumindest die Alphabetisierung. Die entschlossene staatliche Förderung des Ausbaus der Universitäten erlaubte die Ausbildung der wissenschaftlichen Eliten im eigenen Land. Die Verbreitung der avancierten technischen Kommunikationsmittel (Telefon, Radio, TV, Internet) über das gesamte Staatsgebiet gewährte sowohl einen Raum für die Entfaltung der Diversität brasilianischer Kultur, wie sie auch gleichzeitig die symbolische und imaginäre Einheit des Landes und der Nation trotz aller Widersprüche und Ungleichheiten festigte. Die kulturellen Traditionen sind jedoch keineswegs nur positiv: In ihnen ist zwar Wissen und Erfahrung gespeichert, aber dieses Wissen kann auch den Charakter des Aberglaubens, ethnischer, geschlechtsspezifischer und religiöser Vorurteile haben und archaische Wertvorstellungen und Handlungsmuster rechtfertigen. In diesem Fall gilt es dann, ihnen im kulturellen Dialog und in kulturellen Verhandlungen zu begegnen. Nur dadurch kann das Gewaltpotential, das der vehemente gesellschaftliche Transformationsprozess birgt, entschärft werden.

Das brasilianische 20. Jahrhundert zeichnete sich nicht nur durch diesen Modernisierungsprozess aus, sondern auch dadurch, dass die unterschiedlichen sozialen Akteure, die vorher aus den öffentlichen kulturellen Räumen ausgeschlossen waren, jetzt in ihnen präsent wurden, und zwar in doppelter Weise: zuerst als Objekte, als Gegenstand von wissenschaftlichen Untersuchungen und literarischen Erzählungen, dann aber auch als Subjekte selbst. Mit dem Naturalismus rückten erstmals die urbanen populären Schichten in das Blickfeld der Literatur, einer Literatur, die vor allem von den Eliten gelesen wurde. Der Modernismus hob die indianische Vergangenheit als brasilianisches Identitätsmerkmal provokativ auf den Schild und griff damit, wenn auch auf ganz andere Weise, den Indianismus eines José de Alencar aus dem 19. Jahrhundert auf. Er dachte dabei jedoch in keinem Moment an die tatsächlich im Land lebende indianische Bevölkerung, ähnlich wie es bereits bei den Indianisten des 19. Jahrhunderts der Fall gewesen war. Mit dem 'Roman der 30er Jahre' (Romance de 30), zu dem Autoren wie Graciliano Ramos, Raquel de Queiroz und José Lins do Rego 
gehören, wurden die Lebensverhältnisse der ländlichen Bevölkerung des Nordostens in die offizielle brasilianische Literatur eingeschrieben, in Romanen, die dann auch in Übersetzungen einem internationalen Publikum zugänglich wurden. Dies gilt auch für diejenigen Romane, die einige Jahre später die Besiedlungsgeschichte des Südens Brasiliens erzählten, vor allem die Trilogie O Tempo e o Vento (1949-1961) von Érico Veríssimo.

Einer der wichtigsten sozialen Akteure, dessen kulturellen Beitrag Silvio Romero in seiner Literaturgeschichte von 1888 zwar theoretisch einräumte, der aber dennoch aus dem offiziellen kulturellen Leben permanent ausgegrenzt wurde, war die Bevölkerung afrikanischer Abstammung. Obwohl ihr Anteil an der Gesamtbevölkerung zu Beginn des 20. Jahrhunderts bei über $50 \%$ lag, wurde ihr im öffentlichen Kulturleben, in der Literatur und in den anderen Künsten praktisch keine Beachtung geschenkt. Hier öffnete sich mit den Romanen Jorge Amados (Suor [Schweiss], Kakao, Jubiabâ), die die Bevölkerung afrikanischer Abstammung erstmals als historisches Subjekt wahrnahmen, ein ganz neues Panorama. Erstmals begegnete der Leser schwarzen Protagonisten, erstmals ihrer Kultur, ihren Lebensgewohnheiten und ihren religiösen Praktiken (Candomblè). Das aber bedeutete notwendigerweise, dass die Mietskasernen, die Hütten, die Bars, die Arbeitsplätze, die Plantagen und der Hafen von Salvador, kurz, die Orte, an denen diese Bevölkerung lebt und ihr Aus- und Einkommen finden muss, zu Schauplätzen von Romanen wurden. Diese Entdeckung der afrikanischen Kultur und ihrer Bedeutung für die brasilianische Alltagskultur durch Jorge Amado geschah zeitgleich mit der Veröffentlichung der bedeutendsten soziologischen Arbeit zu diesem Thema: Gilberto Freyres Herrenhaus und Sklavenbütte (1933). Auch der aus Recife stammende Soziologe hob in seinem Buch auf den entscheidenden Anteil ab, den die afrikanischen Traditionen für den brasilianischen Alltag haben. Aber bei ihm blieb der portugiesische Kolonisator und später dann der weiße Herr und Arbeitgeber das historische Subjekt, auch wenn er tiefgreifend von afrikanischem kulturellem Erbe verändert wurde. Jorge Amados Sicht, in dessen 'proletarischen' Romanen die schwarze Bevölkerung zum historischen Subjekt wurde, stand dazu in krassem Gegensatz. In seinen Geschichten erzählte er, wie in dieser schwarzen Bevölkerung, die nach der klassischen kommunistischen Theorie zum Lumpenproletariat gehört, ein politisches Klassenbewusstsein entstehen kann.

Gleichzeitig sind diese Romane ein Beleg für die Aufwertung der Populärkultur und machen deutlich, welche Bedeutung ihr für brasilianische 
Kultur überhaupt zukommt, die bis dahin vor allem als Kultur der Eliten begriffen wurde. Eine damals nicht vorhersehbare 'Spätfolge' dieses Perspektivenwechsels ist es, wenn Brasilien heute in den internationalen Medien vor allem durch seine Populärkultur, durch seine Fest- und Musikkultur präsent ist. Aber auch für das kulturelle Selbstverständnis von Brasilien insgesamt spielt Populärkultur eine kaum zu überschätzende Rolle. Es wird durch sie ein 'kulturelles Feld' geschaffen, das für das Zusammenleben entscheidend ist, da es Wertvorstellungen und Urteilskriterien für das Imaginäre seiner kollektiven Subjekte schafft und festigt. Intellektuelle, wirtschaftliche und politische Eliten sind dabei - und das ist gerade wichtig - von Populärkultur nicht ausgeschlossen, sondern suchen oftmals sogar ihre Nähe. Allerdings sind sie in der Regel nicht diejenigen, die Populärkultur hervorbringen, sondern diejenigen, die sie verwerten und politisch oder ideologisch einsetzen. Ihren Sitz, den Ort ihrer Entstehung und ihrer Ausübung hat sie in den sozialen, ethnischen und genderdefinierten Gruppen. Die Subjekte der Populärkultur sind kollektive und keine individuellen Subjekte. Dort, wo individuelle Subjekte an ihrer Realisierung und Ausübung beteiligt sind, tun sie dies immer im Verweis auf und unter Legitimation durch das kollektive Subjekt, nicht als Ausdruck oder Beweis ihrer eigenen individuellen Fähigkeit oder Genialität. Populärkultur zieht sich quer durch die brasilianische Gesellschaft, in ihr werden ihre Widersprüche und Konflikte benannt und semantisiert. Dabei bleibt sie jedoch sehr heterogen: sie ist konservativ und innovativ, ausschließend, indem sie Gruppenidentitäten schafft und sie von anderen abgrenzt, und einschlieBend, indem sie Materialien und Ideen aus allen Bereichen verarbeitet. Sie ist Ort der selbstbewussten Selbstbestimmung, eines empowerment, und Ort der kompromissbereiten, aber auch listigen Verhandlung. Ihre Verwobenheit mit allen gesellschaftlichen Bereichen quer durch Raum und Zeit und die ihr besondere Struktur machen sie zu einem privilegierten Medium lokaler, regionaler oder auch nationaler Identitäten. Gleichzeitig ist sie ein Medium, in dem sowohl 'Verhandlungen' wie auch Auseinandersetzungen zwischen den unterschiedlichsten politischen Akteuren stattfinden. Das gilt zum Beispiel für die politischen und sozialen Bewegungen der Bevölkerung afrikanischer Abstammung (die Quilombola-Bewegungen) und ihre symbolische Aufwertung des afrikanischen Helden Zumbi, der religiösen Praktiken des Candomblé und dem Ritual der Capoeira. Das gilt für die Gender-Bewegungen und auf etwas differenzierte Weise für die Vielzahl der neuen Manifestationen von Religiosität: an die Stelle der noch 
aus der Kolonialzeit stammenden synkretistischen Praktiken, bei denen indianische, an vielen Orten auch afrikanische Traditionen von der katholischen Kirche integriert wurden, treten heute neue religiöse Bewegungen: eine Vielzahl von freikirchlichen Gruppen, einige aus der Pfingstbewegung, aber auch Sekten im eigentlichen Sinne, die sich weitgehend selbst organisieren und finanzieren. Von ihnen werden nicht nur fundamentalistische Glaubensdoktrinen gepredigt, sondern - und hierin liegen ihre Stärke und ihr Erfolg - sie wirken als neue moralisierende Instanz gegen die drohende Anomie, die Gesetzeslosigkeit und den Werteverlust, denen die Bevölkerung vor allem aus der Unterschicht und der unteren Mittelschicht ausgesetzt ist. So sind die Glaubensüberzeugungen mit ganz praktischen Konsequenzen für das Alltagsleben verbunden: Alkohol- und Drogenverbot, keine sexuellen Beziehungen vor der Ehe, Sparsamkeit und Vergnügungsfeindlichkeit (strenge Kleiderordnung, oftmals Tanzverbot). Es geht eine neue moralisierende und ordnende Kraft von ihnen aus, die Parallelen mit dem puritanischen Charakter der Freikirchen im sich industrialisierenden Europa (Methodisten, Adventisten, Baptisten, etc.) hat.

Diese 'marginalisierten' Räume der Populärkultur, die im Grunde die ausgedehntesten in der brasilianischen Gesellschaften sind, denn 'marginal' ist eigentlich eher die offizielle Kultur, sind also nicht nur Objekte für die Kulturproduktion der Eliten (Spiel- und Dokumentarfilme, z. B. der Film Cidade de Deus), für Großstadtromane oder Genderromane, ihre Bewohner werden nicht nur als Konsumenten einer florierenden Massenkultur und ihrer Kulturindustrie entdeckt (vor allem der Musikindustrie und als Publikum der Telenovelas), sondern sie werden - notwendigerweise - auch zu selbstständigen Kulturproduzenten, in dem Sinne, dass selbstorganisierte Alltagspraxis ohne Kultur nicht denkbar ist. Der Übergang von Populärkultur zu Massenkultur ist durchaus fließend: Populärkultur wird zu einem festen Bestandteil der Kulturindustrie, wie auch im Gegenzug Elemente der Kulturindustrie von der Populärkultur angeeignet und transformiert werden. Vereinfacht und schematisch kann man behaupten, die Subjekte der Populärkultur sind die kollektiven Subjekte der unterschiedlichen kulturellen Akteure, das Subjekt der Kulturindustrie und der Massenkultur ist das Kapital und die Bedingungen seiner Verwertung, der finanzielle Gewinn.

Waren das gesamte 19. Jahrhundert hindurch die ländlichen Räume der privilegierte Ort von Populärkultur, so sind es im Laufe des 20. Jahrhunderts die Städte und die urbanen Agglomerationen geworden. Modernisierung bedeutet in Brasilien wie auch in anderen Ländern die Umstruk- 
turierung der Gesellschaft in eine vorwiegend urbane Gesellschaft. Wie sehr Modernisierung in all ihrer Widersprüchlichkeit und Ambivalenz mit Stadtkultur verbunden ist, zeigen wahrscheinlich wenige Ereignisse klarer als der Entschluss in den 1950er Jahren, Brasilien in seinem unbesiedelten Inneren, auf dem Altiplano, eine neue Hauptstadt zu geben, und die tatsächliche Realisierung dieses Entschlusses durch die Einweihung dieser neuen Hauptstadt, Brasília, im Jahre 1960. Der Architekt Oscar Niemeyer und der Stadtplaner Lúcia Costa entwarfen eine Stadt, die den Idealen einer modernen demokratischen Gesellschaft entsprach, sie konstruierten mit den avanciertesten Techniken und mit einer Ästhetik, die in nichts an brasilianischen Barock oder an lokale Bautraditionen erinnerte, sondern sich ganz einer universellen Moderne verschrieb.

Dieser neue Regierungssitz wurde vier Jahre später nach einem Staatsstreich der Militärs zum Sitz der Militärregierung, die Brasilien mehr als 20 Jahre lang beherrschte. So wie die demokratische Utopie eines modernen Brasiliens den ebenso modernen Panzern der Militärs zum Opfer fiel, die in Rio de Janeiro und São Paulo jeden Widerstand gegen den Putsch brutal unterdrückten, so zeigte auch der allgemeine Modernisierungsprozess, der Brasilien in den folgenden Jahrzehnten zur führenden Nation Südamerikas werden ließ, ein Janusgesicht: modernste Industrieanlagen und Favelas, Zunahme des Wohlstandes und Ausgrenzung großer Bevölkerungsteile, Universitäten und Forschungseinrichtungen auf international vergleichbarem Niveau und unkontrollierbare Gewalt in den Vorstädten, Körperkult und höchster Standard in plastischer Schönheitschirurgie und prekäre gesundheitliche Versorgung der armen Bevölkerung. Diese Liste könnte noch lange fortgesetzt werden.

Literatur war immer ein Medium, in dem Erwartungen wie auch Kritiken sehr sensibel registriert wurden. Das gilt auch für die brasilianische Literatur in der zweiten Hälfte des 20. Jahrhunderts. Obwohl ihr im Gegensatz zu den hispanoamerikanischen Literaturen, die im Laufe des 20. Jahrhunderts vier Nobelpreise zugesprochen bekamen, kein solcher Preis verliehen wurde, sind ihre Autoren dennoch bei einem internationalen Lesepublikum bekannt geworden. Jorge Amado ist bis heute der meist übersetzte brasilianische Autor, wenn ihn in den letzten Jahren auch Paulo Coelho an Auflagenzahlen übertroffen hat. Das Gewicht der regionalen Literaturen nimmt in der brasilianischen Literaturlandschaft der zweiten Hälfte des letzten Jahrhunderts zu. Der Nordosten, der Sertão, wird mit Guimarães Rosa und Grande Sertão, Veredas (1956) in die Weltliteratur 
eingeschrieben, der Süden ist mit Moacyr Scliar, João Gilberto Noll und vielen jungen AutorInnen präsent, Amazonien mit Márcio Souza und Milton Hatoum. Das sind einige eher beliebig ausgewählte Namen, zu denen noch eine große Anzahl weiterer hinzuzufügen wäre. Die Mehrzahl der Autoren und Autorinnen aber, die sowohl im nationalen Kontext wie auch in der internationalen Wahrnehmung Gewicht haben, schreiben über die Wirklichkeiten der Städte, allen voran die der Städte Rio de Janeiro und São Paulo. Clarice Lispectors letzter Roman Sternstunde (1977) hat als Protagonisten eine in Rio de Janeiro verlorene Migrantin aus dem Nordosten und den Erzähler, Rodrigo, selbst. Er schreibt über eine Welt, die nicht die seine ist. Damit steht er vor dem Problem, das sich immer stellt, wenn sich die Kultur der Elite vornimmt, Figuren, Lebensweise und Konflikte einer Bevölkerungsschicht zu repräsentieren, mit der sie keinerlei Erfahrung teilt. Der Erzähler des Romans fragt sich, was ihm überhaupt das Recht gibt, über die Erfahrung dieser einfachen Frau aus dem Nordosten in Rio de Janeiro zu schreiben, auch wenn sie selbst keine Worte dafür hat, selbst niemanden kennt, der ihr zuhören könnte und darüber hinaus überzeugt ist, ihr Leben sei sowieso ohne jegliche Bedeutung. Auch in Ignacio Loyola Brandãos ersten Stadtromanen Null (1974) und Kein Land wie dieses (1982) sind die Protagonisten hilflos der modernen Stadt und ihren unmenschlichen Lebensbedingungen ausgeliefert. Diese Opferperspektive wird in den Erzählungen und Romanen Rubem Fonsecas abgelehnt. Bei ihm werden aus den Opfern Täter. Dieser Umschlag, die Identifikation des Erzählers mit den Tätern, ist für den größten Teil der neueren Stadtliteratur, wie z. B. für die Romane von Patricia Melo (O matador, 1995; Inferno, 2000) symptomatisch. Problematisch bleibt dabei allerdings, was Clarice Lispector bereits im Falle ihres Erzählers Rodrigo thematisierte: wodurch rechtfertigt sich diese Einfühlung in den Täter? Welche Glaubwürdigkeit kommt ihr zu? Findet hier nicht im Grunde eine literarische Ausbeutung der Marginalisierten statt? Paulo Lins, dessen Roman Cidade de Deus (1997) in seiner verfilmten Fassung City of God (Fernando Meirelles und Kátia Lund) zu einem Welterfolg wurde, rechtfertigt sein Schreiben durch seine Arbeit als Anthropologe in der Favela, die Schauplatz des Romans ist.

Die autobiographischen Texte eines Luiz Alberto Mendes (Memórias de um sobrevivente, 2001; Às Cegas, 2005) sind nicht nur aus der Perspektive der Täter, sondern von einem Täter selbst geschrieben. Der Autor schrieb diese Autobiographie, in der er seinen Weg vom einfachen Dieb zum Mörder erzählt, im Gefängnis, in dem er seine Haft für Überfälle und 
Mord zu verbringen hatte. Das Praktische Handbuch des Hasses (2003) von Ferréz (Reginaldo Ferreira da Silva) ist einer der Titel, in denen die Welt der Favelas der Schauplatz ist, die Welt, in der er selbst seine Jugend verbracht hat und in der er auch heute noch lebt. Das gleiche gilt für die Texte von MV Bill und Celso Athayde (Falcão - Kinder des Drogenhandels, 2006). Ihre Zuordnung schwankt zwischen Zeugentext und Autofiktion. Auch sie zeichnet ein extremer Realismus der Gewalt mit dem täglichen Kampf ums Überleben als zentrales Thema aus.

Das Medium Literatur kann sich einen Realismus erlauben, der dem Medium Film und mehr noch der Telenovela, jenem Medium, mit dem Brasilien Welterfolge feiert, nicht erlaubt ist. So endet der bereits erwähnte Film City of God relativ versöhnlich, indem der Protagonist Buscapé die Übergriffe der Polizei dokumentieren kann und ihm dafür eine aussichtsreiche Berufskarriere in Aussicht steht. Der Roman selbst schließt mit einem unerbittlichen Bandenkrieg. Dennoch ist urbane Gewalt auch eines der favorisierten Themen im Brasilianischen Kino der Gegenwart. In Onibus 174 (2002) von José Padilha wird die Entführung eines Omnibusses rekonstruiert, Carandirú (2003) von Hector Babenco verfilmt den Bericht des Arztes Dráuzio Varella über eine Gefängnisrevolte in São Paulo im Jahr 1992. Tropa de Elite (2007) (José Padilha) ist ein Film über eine Spezialeinheit der Polizei und deren Kampf gegen den Drogenhandel. Die alltägliche Gewalt wird nicht nur von den internationalen Medien als eines der großen Probleme des Landes wahrgenommen, auch die brasilianischen Medien selbst diagnostizieren sie als eine der größten Herausforderungen zu Beginn des 21. Jahrhunderts.

Wenn wir zu Beginn der Literatur des 19. Jahrhunderts eine entscheidende Rolle bei der Herausbildung nationaler Identität zugewiesen und ihren Beitrag betont haben, eine imaginäre Repräsentanz zu schaffen, was und wie Brasilien sein soll, so lässt sich behaupten, dass diese Aufgabe seit den 1980er Jahren des 20. Jahrhunderts von den Telenovelas übernommen worden ist. Die medialen und technischen Voraussetzungen dafür hat das Rede Globo geschaffen, heute die drittgrößte Fernsehanstalt der Welt. In den Telenovelas bespiegelt sich die brasilianische Gesellschaft auf eine gut konsumierbare Art und Weise. Mit etwas Selbstkritik, mit einer guten Portion Problembewusstsein, auch mit Selbstironie. Meist sind es Liebesgeschichten mit glücklichem Ausgang, oftmals auch mit einem sozialen Aufstieg für das 'Aschenputtel' verbunden, das zwar arm aber von edlem Charakter ist. Gleichzeitig haben sie ein gewisses 'Aufklärungspo- 
tential': Das indirekte Lob der Kleinfamilie soll das weibliche Publikum daran erinnern, dass ein sozialer Aufstieg für eine kinderreiche Familie oder gar eine alleinstehende Frau mit mehreren Kindern undenkbar ist. Helden afrikanischer Abstammung sind seit langem mit den gleichen Tugenden ausgestattet wie ihre weißen Kollegen, der Bösewicht wird nicht mehr durch seine ethnische Zugehörigkeit definiert. In vielen Serien hat auch ein Gay oder eine Lesbe einen ehrenwerten Part. Wie groß die moralisierende Wirkung dieser Telenovelas letztendlich ist, lässt sich nur schwer feststellen. Letztlich besteht ihre Funktion eben doch darin, ein unterhaltsames Programm zu bieten, das außerdem nicht nur auf Brasilien ausgerichtet ist, da dies den Export, vor allem auf den hispanoamerikanischen Markt, beeinträchtigen würde. Allerdings wurden in den letzten Jahren auch Telenovelas produziert, die ganz explizit auf einen internationalen Markt ausgelegt sind, gedreht auf internationalen Schauplätzen (Nordafrika, Indien, China) mit historisch-exotischen Handlungsführungen. O Clone (2001-2002) (Gloria Pérez) und Caminhos da Índia (2009) (Carlos Lombardi) sind Beispiele dafür.

In der zweiten Hälfte des 20. Jahrhunderts fand Brasilien endgültig seinen Platz auf der Weltbühne, wirtschaftlich, politisch und kulturell. Auf diesem durch Medien determinierten Schauplatz spielen auch fünf gewonnene Fußballweltmeisterschaften eine nicht zu unterschätzende Rolle. Aber wer erinnert sich heute noch daran, dass das Land 1970 die Fußballweltmeisterschaft gewann, genauso wenig wie wir uns daran erinnern, dass dies gerade die härtesten Jahre der Militärdiktatur waren und gleichzeitig auch die Jahre der deutsch-brasilianischen Verhandlungen über ein gemeinsames Programm für den Bau des Atommeilers in Ancra. Woran wir uns nicht erinnern müssen, weil wir sie an den unterschiedlichsten Orten der Welt plötzlich hören, sind die Klänge der Música Popular Brasileira, diese Mischung aus Bossa Nova, Samba und Jazz, die uns so unvergleichlich brasilianisch anmutet. Die Namen von Vinicio de Moraes, Gilberto Gil, Caetano Veloso, Chico Buarque, Elis Regina und vieler anderer aus dieser Musikszene sind länger im Gedächtnis geblieben als die Namen der Generäle der Diktatur.

Ob der Ausspruch, Brasilien sei kein ernst zu nehmendes Land, wirklich dem damaligen französischen Präsidenten de Gaulle zuzuschreiben sei, mag dahin gestellt sein. Sicher aber ist, dass es heute an der Ernsthaftigkeit Brasiliens keinen Zweifel mehr gibt. Seine politische Rolle in Amerika, die Konkurrenzfähigkeit seiner Technologien und seiner Industrie und sein 
Entwicklungspotential haben es zu einem der fünf BRICS-Staaten werden lassen. Dass eine solche Entwicklung möglich war in einem Land, das vor weniger als 200 Jahren seine Unabhängigkeit erklärte, in dem vor 125 Jahren die Sklaverei abgeschafft wurde, das im 20. Jahrhundert durch zwei lange Diktaturperioden (der Estado Novo von Getulio Vargas (1937-1945) und die Militärdiktatur (1964-1985) hindurch ging, ist nicht zuletzt auch seiner kulturellen Dynamik zuzuschreiben.

\section{Literaturverzeichnis}

Andrade, Oswald de (1972): Do Pau Brasil à Antropofagia e às Utopias: manifestos, teses de concursos e esaios. Rio de Janeiro: Civilização Brasileira.

Birle, Peter/Costa, Sérgio/Nitschack, Horst (Hg.) (2008): Brazil and the Americas. Convergences and Perspectives. Madrid/Frankfurt am Main: Iberoamericana/Vervuert.

Costa, Sérgio/Kohlhepp, Gerd/Nitschack, Horst/Sangmeister, Hartmut (Hg.) (2010): Brasilien heute. Geographischer Raum, Politik, Wirtschaft, Kultur. 2. vollständig neu bearbeitete Auflage. Frankfurt am Main: Vervuert.

CunHA, Euclides da (1994): Krieg im Sertão. Übersetzt von Berthold Zilly. Frankfurt am Main: Suhrkamp.

DaMatta, Roberto ([1984] o.J.): O que fazo o brasil, Brasil. Rio de Janeiro: Rocco.

- ([1985] 51997): A Casa \& a Rua. Espaço, Cidadania, Mulber e Morte no Brasil. Rio de Janeiro: Rocco.

FREYRE, Gilberto (1965): Herrenhaus und Sklavenhütte: ein Bild der brasilianischen Gesellschaft. Übersetzt von Ludwig Graf von Schönfeldt. Köln/Berlin: Kiepenheuer \& Witsch.

Holanda, Sérgio Buarque de ([1936] $\left.{ }^{13} 1979\right)$ : Raízes do Brasil. Rio de Janeiro: Olympio.

Nitschack, Horst (2011): "Subjetividade e violência no romance urbano atual do Brasil". In: Sartingen, Kathrin/Ugalde, Esther Gimeno (Hg.): Perspectivas actuais na Lusitanística. München: Martin Meidenbauer, 13-26.

Ortiz, Renato ([1985] 1994): Cultura Brasileira \& Identidade Nacional. São Paulo: Brasiliense.

Romero, Silvio ([1888] ${ }^{71980): ~ H i s t o ́ r i a ~ d a ~ l i t e r a t u r a ~ b r a s i l e i r a . ~} 5$ Bde., Rio de Janerio/Brasília: Livraria José Olympio Editora in Zusammenarbeit mit Instituto Nacional do Livro/ Ministério da Educação e Cultura. 